\title{
FACTORES EXPLICATIVOS DE LA DESERCIÓN UNIVERSITARIA
}

\section{Introducción}

La educación superior (ES) chilena ha experimentado cambios significativos durante los últimos veinte años. Durante este periodo se produjo una fuerte expansión en el acceso al sistema educativo, impulsada principalmente por el incremento en la matrícula universitaria. Según cifras del Consejo Superior de Educación (CSE) las universidades absorben cerca del 70\% de la demanda de educación superior (CSE, 2006). En contraposición, los centros de formación técnica (CFT) e institutos profesionales (IP) constituyen una proporción cada vez menor de la matrícula total del sistema terciario.

Una de las razones que explican el incremento de la matrícula terciaria es la fuerte expansión de las expectativas de la población en la ES. Cifras aportadas por la Encuesta Nacional de Actores del Sistema Educativo dan cuenta de esta expansión en todos los grupos socioeconómicos de la sociedad chilena (CIDE, 2001, 2003). La creciente aspiración por ingresar a la ES se explica, en parte, por los altos retornos monetarios que conlleva el paso por el sistema. En el caso de Chile, los retornos de la educación universitaria se estiman en un $21 \%$, cifra bastante superior a los obtenidos por profesionales de países miembros de la $\mathrm{OECD}^{1}$, que sólo llegan a un promedio de 12\% (Psacharopoulos, 2002). Por lo tanto, el acceso a la ES representa la posibilidad de obtener ingresos muy superiores a los de quienes no ingresan a ella.

Producto del incremento de expectativas y de las altas tasas de retorno monetario, nuestro país está experimentando,

1 Organization for Economic Co-operation and Development (OECD). 
desde hace algunos años, un proceso de creciente masificación de esta clase de educación. Esto ha traído como consecuencia una mayor heterogeneidad en el sistema, que se manifiesta en una mayor diversidad de instituciones proveedoras y, también, en una composición socioeconómica y cultural más heterogénea del alumnado que ha ingresado durante los últimos años. Las cifras indican que, en 1990, más del 40\% de los estudiantes que cursaban estudios superiores pertenecían al quintil más rico de la población, mientras en 2000 esta cifra disminuyó a un tercio (Delannoy, 2000). Adicionalmente, las cifras provistas por el INE (2003) muestran que alrededor de un $70 \%$ de los alumnos que ingresan hoy son la primera generación de sus familias en cursar estudios superiores.

Sin embargo, a pesar que la cobertura ha aumentado significativamente, la proporción de individuos que la finaliza es relativamente pequeña. En 2005, si bien la matrícula bruta de ES alcanzaba al $42 \%$ de la cohorte, sólo un 13\% de la población chilena entre 25 y 64 años contaba con estudios superiores completos (OECD, 2005; World Bank, 2005). Esto quiere decir que sólo uno de cada diez chilenos había completado una carrera en la ES.

Lo anterior implica que, las tasas de retención y graduación son relativamente bajas respecto de la proporción total de estudiantes que ingresa al sistema. Los datos de retención y eficiencia para el sistema educativo chileno son concordantes con este diagnóstico e indican que un significativo número de estudiantes abandona su carrera en el primer o segundo año, siendo menor la proporción de estudiantes que desertan a medida que se avanza en el sistema educativo superior (Himmel, 2002). La información disponible muestra que uno de cinco estudiantes abandonaba la carrera antes del primer año y, en segundo año, sólo dos de tres permanecía estudiando la carrera a la cual ingresaron inicialmente (CSE, 2007). Otras estimaciones indican que sólo un tercio de los alumnos que permanecen más allá del segundo año se gradúa oportunamente, estando esta cifra por sobre las tasas exhibidas en países de la OECD (OECD, 1998, 2000, 2002, 2004). 


\section{Antecedentes bibliográficos}

Escasas investigaciones exploran el fenómeno de la deserción universitaria desde una perspectiva explicativa. Los estudios han estado orientados a generar estimaciones cuantitativas sobre su magnitud y sus costos y, en menor medida, entender las condiciones que desencadenan los procesos de deserción.

La literatura internacional, por el contrario, es extensa y variada en el tema. Gran parte de los estudios muestran que existen diversas formas de comprenderla, así como también los distintos tipos de deserción, que varían de acuerdo con las causas que originan el fenómeno. Los estudios que se presentan a continuación han sido seleccionados porque constituyen estudios fundacionales ${ }^{2}$ en el tema o, bien, porque resultan especialmente atingentes para analizar la deserción en nuestro país. Para comenzar, se presentan algunas definiciones básicas del concepto "deserción en la educación superior".

En el ámbito internacional, Tinto ha sido uno de los investigadores que más ha contribuido a la comprensión del fenómeno. En su trabajo, desarrollado para Estados Unidos, introduce una interesante discusión conceptual acerca de la deserción, en relación con la naturaleza voluntaria u obligatoria del fenómeno, así como también el carácter permanente o temporal del proceso. Este autor comprende la deserción (1975) como el proceso de abandono voluntario o forzoso de la carrera en que se matricula un estudiante, por la influencia positiva o negativa de circunstancias internas o externas al alumno.

Tinto plantea que el abandono de los estudios superiores puede tener carácter tanto transitorio y/o permanente, de acuerdo con el tiempo que dure el alejamiento del estudiante de las instituciones de ES. La deserción es transitoria, cuando el alumno decide revertir la situación de abandono, en la medida de que éste no tenga impedimento para retomar sus estudios; se torna permanente cuando

2 Estos estudios son frecuentemente citados por los investigadores en el área. 
existen causas concretas que impiden al alumno volver a cursar o retomar sus estudios superiores.

En nuestro país, Himmel (2002) ha desarrollado una determinación diferente del concepto. Esta investigadora define la deserción como "el abandono prematuro de un programa de estudios antes de alcanzar el título o grado, y considera un tiempo suficientemente largo como para descartar la posibilidad de que el estudiante se reincorpore" (2002; 95). En relación con la naturaleza voluntaria u obligatoria del proceso, Himmel señala que la deserción voluntaria puede entenderse como la renuncia a la carrera por parte del estudiante o como un abandono no informado a la institución. La no voluntaria, en cambio, se produciría como consecuencia de una decisión institucional, fundada en reglamentos universitarios que obligan al alumno a retirarse de su carrera (Himmel, 2002) . $^{3}$ En su visión, la decisión de desertar hay que entenderla como un proceso que ocurre a través del tiempo y no como una situación detonada en un punto discreto. Himmel agrega un nuevo elemento a la discusión, señalando que el abandono difiere si se realiza de una carrera (programa) o de una institución. La deserción de una carrera no implica necesariamente la segunda, debido a que el estudiante puede abandonar una carrera e ingresar a un programa diferente dentro de la misma institución, ya sea por transferencia o por reingreso a través del proceso de admisión ordinaria. La deserción de una institución, mientras tanto, puede significar transferencia a un programa en otra institución o el abandono definitivo de la ES (Himmel, 2002).

Estas definiciones dan cuenta de la complejidad y variabilidad del fenómeno, y de la existencia de matices y de distintos tipos de deserción, difíciles de tipificar en la práctica.

En relación con las causas que producen la deserción, las investigaciones muestran que no es posible atribuir la deserción a

3 Himmel identifica como causas de deserción involuntaria el deficiente desempeño académico o razones disciplinarias. 
una sola sino, más bien, a una multiplicidad de factores. Entre los destacados por diversos investigadores se encuentran, por un lado, factores personales, culturales, sociales y económicos de los alumnos y sus familias, y, por otro, factores académicos e institucionales. Parece no existir consenso respecto de si las causas individuales, sociales o académicas son las más determinantes a la hora de explicar la deserción.

Por ejemplo, Tinto (1975) plantea que la deserción es causada por la interacción de elementos individuales, sociales e institucionales. En relación con los aspectos sociales, el autor destaca la centralidad de las características o antecedentes familiares de los estudiantes, siendo especialmente significativos aquellos relacionados con la ocupación y nivel educacional de los padres, la valoración y expectativas educativas de los jóvenes y el compromiso con una meta de estudios y con futuros objetivos.

Concordante con esto, Bank, Biddle y Slavings (1990) destacan la influencia de los padres y los pares en las expectativas que los alumnos tienen sobre la ES. Si los pares y padres tienen actitudes positivas hacia la educación, existen menos probabilidades de que los alumnos deserten del sistema.

Bean y Metzner (1985) también destacan la importancia de las variables de contexto social y económico de los alumnos como causas de deserción. Entre los factores más relevantes consideran la situación financiera del estudiante y su familia, trabajo (horas de empleo) y las responsabilidades familiares, entre otros. Señalan que estos aspectos son muy significativos para alumnos que provienen de grupos sociales que se han incorporado recientemente a la ES. Las variables académicas, por el contrario, parecen ser más relevantes en los casos de alumnos "tradicionales" del sistema, es decir, que provienen de familias con estudios superiores.

Por otra parte, Reed (1968) analiza la relevancia de los factores motivacionales en el proceso de deserción, especialmente en la deserción 
de carácter voluntario. Señala que existe una importante asociación entre la motivación del estudiante y sus rendimientos académicos. Las variables que incidirían en la motivación del alumnado serían el sentido que el alumno asigna a las tareas académicas, la relevancia de los estudios en relación con sus metas futuras, el desafío académico de la carrera, entre otros aspectos.

Reay, Davies y Ball (2001) señalan que la deserción tiene relación con el acceso de los estudiantes a la ES. Sostienen que el proceso que lleva a tomar la decisión de ingresar es una manifestación de poder y estratificación. Los postulantes tienen distintos tipos de oportunidades para elegir una carrera o institución, las cuales se ven determinadas por particulares circunstancias y restricciones. Factores como aranceles, préstamos y becas, ubicación (cercanía-lejanía del hogar), clase y raza de los alumnos son variables que limitan las oportunidades de elección y permanencia en el sistema terciario. En general, los estudiantes de clase baja deciden ingresar a instituciones educativas en las cuales piensan que se encontrarán con gente similar a ellos, se sentirán más a gusto o menos excluidos. Esta situación se explica porque los alumnos tienen un sentido de lugar y de mundo asociado al lugar que ellos ocupan dentro del espacio social.

En relación con los factores institucionales y/o académicos que explican la deserción, Spady (1970) y Tinto (1975) mencionan la falta de integración del estudiante a las instituciones educativas como un aspecto de gran relevancia. En términos generales, sugieren que los alumnos abandonan las instituciones cuando ellos no se sienten integrados al sistema académico y social de éstas. Ambos desarrollan sus enfoques tomando como punto de partida el trabajo de Durkheim (1951) sobre el suicidio, indicando que la deserción debe ser entendida como un fenómeno de desintegración ${ }^{4}$.

4 Durkheim sostiene que el suicidio es el resultado de la ruptura del individuo con el sistema social por su imposibilidad de integrarse a la sociedad. La posibilidad de suicidio aumenta cuando existe una baja conciencia moral y los individuos tienen escaso apoyo de las relaciones sociales 
Señalan que las instituciones educativas deben comprenderse como sistemas sociales con valores y estructuras. Cuando los alumnos no comparten los sistemas de creencias o valores de las instituciones donde estudian sufren falta de integración o escaso sentido de pertenencia, pudiendo generar un bajo compromiso con sus estudios, lo que incrementa la probabilidad de abandonar o desarrollar actividades alternativas. Ambos autores distinguen dos formas de integración en las instituciones educativas: académica y social. Los factores que se relacionan con la integración académica del estudiante se vinculan con el rendimiento académico y su desarrollo intelectual; la integración social, en cambio, se relaciona con las oportunidades de interactuar con pares y docentes ${ }^{5}$ y también con la oferta y participación de los alumnos en actividades extracurriculares.

De manera complementaria al trabajo de Tinto y Spady, Tierney (1999) utiliza el concepto de habitus de Bourdieu (1973, 1986) para analizar cómo las prácticas sociales desarrolladas en las instituciones educativas se relacionan con aspectos sociales y culturales más amplios que trascienden la vida académica como tal. Señala que, a medida que los estudiantes son capaces de afirmar sus identidades culturales en las instituciones educativas, sus posibilidades de graduarse también aumentan.

La visión de Tierney sugiere que en el proceso de deserción no sólo el estudiante es el responsable, sino que también la organización académica juega un rol importante. Light y Strayer (2000) concuerdan con los planteamientos de Tierney, al señalar que la deserción está muy determinada por la calidad de las instituciones de ES y los soportes que éstas brindan a los estudiantes. Braxton, Johnson y Shaw-Sullivan (1997) profundizan en el tema de la calidad de las instituciones, señalando que los factores asociados a la calidad de la docencia, los beneficios (apoyos académicos y de salud) y las actividades complementarias (actividades culturales y deportes), así como las

5 Este factor parece ser mucho más importante y valorado por los estudiantes de clases sociales medias y altas. 
experiencias de los estudiantes en el aula, juegan un rol central en su retención o deserción. Tillman (2002), por su parte, sostiene que la disponibilidad de recursos bibliográficos, de laboratorios, así como también el número de alumnos por profesor, son variables significativas que dan cuenta de la calidad de las instituciones y que influyen en la decisión de deserción. Fielding, Belfield y Thomas (1998) mencionan también el proceso de enseñanza y los profesores como aspectos claves que explican el abandono o retención.

En nuestro país, los estudios sobre deserción han coincidido con la evidencia internacional respecto a la multicausalidad del fenómeno. González y Uribe (2005) afirman que factores institucionales y aspectos sociales son causantes de la deserción en el nivel terciario. Entre los factores más relevantes mencionan que las instituciones no se han adaptado al nuevo tipo de alumnos que ingresa al sistema, la insuficiente o mala calidad de la información que reciben los estudiantes para elegir una carrera, la extensa duración de éstas, las razones vocacionales, motivacionales, económicas, o una combinación de estas causas.

Al igual que González y Uribe, Himmel (2002) destaca la interacción de aspectos individuales, sociales e institucionales como detonantes de la deserción universitaria. Entre las causas sociales e individuales menciona los valores y expectativas personales y familiares, el autoconcepto académico de los alumnos, los referentes familiares de los alumnos, entre los más importantes. En relación con los factores de tipo institucional, destaca la falta de integración de los estudiantes a sus instituciones, los problemas de calidad de la docencia y los sistemas de apoyo institucional.

Dos estudios, que no se abocan directamente a tratar el tema de la deserción pero que se centran en los problemas de la ES chilena, sugieren algunas hipótesis interesantes en torno a las causas de abandono. Un estudio del Programa de las Naciones Unidas para el Desarrollo (2005) sugiere que los factores socioeconómicos parecieran incidir en el proceso de deserción. A partir de un análisis de la encuesta 
CASEN (2003), este estudio establece que la deserción en jóvenes de familias acomodadas es menos frecuente que en los casos de alumnos que provienen de estratos sociales menos pudientes.

Por otra parte, Brunner y Meller (2004) plantean que las bajas tasas de eficiencia ${ }^{6}$ del sistema educativo terciario podrían explicarse por factores asociados con las instituciones educativas y características propias del sistema. Mencionan la falta de flexibilidad curricular de las carreras, la ausencia de sistemas adecuados de progresión y problemas en los procesos de selección de estudiantes como aspectos que explican los magros resultados en comparación con otros sistemas de ES en el mundo.

\section{Metodología del estudio}

En esta investigación, la deserción se definió como el proceso de abandono voluntario o forzoso de una carrera, proceso que puede asumir un carácter transitorio o permanente, dependiendo del tiempo y de los obstáculos de reinserción. Se decidió explorar los factores explicativos de la deserción temporal y permanente en la educación universitaria actual. Como objetivo más específico, se intentó identificar los factores académicos y extraacadémicos que inciden en las dos formas de deserción previamente descritas.

Se consideró como desertores universitarios temporales a aquellos estudiantes que abandonaron al menos una vez una carrera universitaria, pero que al momento de llevar a cabo esta investigación se encontraban estudiando una nueva carrera en la misma u otra universidad. Por otra parte, se definió a los desertores permanentes como aquellos estudiantes que se encontraban alejados de la educación superior formal al momento de realizar este estudio.

6 Si bien este estudio no se centra en el tema de la deserción, puede considerarse una aproximación al tema, ya que se analizan las causas que explican las bajas tasas de eficiencia de la educación superior en Chile, lo cual tiene relación con las menores tasas de graduación y los problemas de repitencia y deserción que experimenta el sistema. 
Hubo contacto con diferentes instituciones de educación superior del país y la entrega de información se concretó con unidades académicas de nueve universidades. Dada la disposición a participar en el estudio, la muestra consideró cuatro universidades privadas y cinco universidades públicas. De estas nueve instituciones, sólo una universidad se encuentra ubicada fuera de la Región Metropolitana, por lo tanto, una proporción mayoritaria de los casos representa la situación de estudiantes de Santiago.

Al contactar a los estudiantes de las universidades participantes de este estudio, se constató que una proporción relevante de desertores temporales habían experimentado múltiples deserciones. Aunque este dato puede ser considerado una debilidad metodológica, también muestra una realidad no claramente anticipada de este estudio y, por otra parte, escasamente estudiada y cuantificada en el país.

Se utilizó la Clasificación Internacional Normalizada de la Educación (CINE) de la $\mathrm{UNESCO}^{7}$ (1997) para definir las áreas del conocimiento en las cuales se seleccionaría a los desertores. La selección de la muestra contempló desertores de las siguientes áreas: Forestales y Agropecuarias, Arte y Arquitectura, Ciencias Exactas y Naturales, Ciencias Sociales, Derecho, Humanidades, Educación, Tecnología y Administración y Comercio ${ }^{8}$.

Los desertores fueron seleccionados a partir de la información de los sistemas de registro curricular de las instituciones o a través de las coordinaciones académicas de las respectivas carreras. Se contactó a los entrevistados telefónicamente o vía e-mail, invitándolos a participar de las entrevistas individuales y grupales. La tasa de respuesta fue de un 20\% aproximadamente, considerado normal para este tipo de estudios.

7 Organización de las Naciones Unidas para la Educación, la Ciencia y la Cultura.

8 Se excluyó el área Salud, porque las cifras existentes sobre deserción en nuestro país, de acuerdo con González y Uribe (2005), indican que en esta área son muy bajas o casi inexistentes. 
En total, se realizaron doce entrevistas en profundidad a desertores permanentes y doce entrevistas grupales a desertores temporales ${ }^{9}$. El total de entrevistados fue de sesenta y siete ${ }^{10}$; de ellos, doce correspondieron a desertores permanentes. Dada la naturaleza cualitativo-exploratoria de este estudio, la selección de casos privilegió la representación de distintas áreas de conocimiento y universidades en la muestra. En las entrevistas grupales ${ }^{11}$ se intentó convocar a estudiantes de universidades y carreras similares con el fin de facilitar el diálogo entre participantes. Tanto las entrevistas individuales como las grupales se realizaron siguiendo una pauta semiestructurada ${ }^{12}$ de entrevista ${ }^{13}$, con dos moderadores ${ }^{14}$. Esta metodología permitió considerar nuevas preguntas sobre temas no contemplados inicialmente.

En el análisis de las entrevistas se utilizó inicialmente un sistema de codificación abierta, que permitió identificar propiedades y conceptos relevantes (Strauss \& Corbin, 1998) asociados a los patrones de deserción. Se empleó una metodología de análisis de casos para analizar por separado las causas de deserción temporal y permanente. El uso de casos variados (diferentes instituciones, localidades y áreas de conocimiento) proporcionó antecedentes que permitieron comprender el contexto y las explicaciones que los estudiantes dan a sus experiencias de deserción.

9 Las entrevistas fueron grabadas previo consentimiento de los participantes. Para asegurar la voluntaria participación, los entrevistados firmaron una carta de consentimiento informado.

10 La edad promedio de los entrevistados fue de veintitrés años, siendo el menor de dieciocho años y la mayor de treinta y nueve. De los entrevistados, cuarenta y cinco fueron hombres y veintiocho mujeres, lo que corresponde a un $62 \%$ y un $38 \%$ de la muestra, respectivamente

11 En promedio, el número de participantes en cada entrevista grupal fue de cinco personas. Este número facilitó la dinámica de conversación y posibilitó profundizar los temas de interés para esta investigación.

12 Esta pauta fue testeada y corregida después de realizar las primeras entrevistas grupales.

13 Para otorgar mayor rigurosidad a las condiciones de recolección de datos, todas las entrevistas grupales fueron desarrolladas con dos moderadores. El moderador principal guió la entrevista y el secundario se encargó de chequear que todos los temas relevantes establecidos en la pauta fueran cubiertos en esta actividad.

14 La pauta de entrevista individual es una versión adaptada de la pauta de entrevista grupal. 


\section{Resultados del estudio}

Los resultados de esta investigación indican que la deserción temporal y permanente son fenómenos diferentes. El análisis de las causas que originan ambos tipos de deserción sugiere que, en ambos casos, las razones no académicas juegan un rol muy relevante, pero los factores específicos que provocan la deserción en ambos tipos de deserción difieren. Específicamente, la evidencia sugiere que, mientras la deserción temporal está fuertemente asociada a causas de tipo vocacional, motivacional y sociocultural, la permanente se explica principalmente por condicionantes socioeconómicas y familiares.

Si bien los factores académico-institucionales resultaron ser menos relevantes de lo anticipado, la investigación mostró algunos hechos que deben ser destacados, con el fin de ayudar a la comprensión de los procesos de deserción en nuestro país. En primer lugar, un mal rendimiento o bajo desempeño académico no es el principal problema entre los factores académicos que explican la deserción, sino que parecen estar fuertemente vinculados a problemas de naturaleza motivacional y vocacional de los estudiantes.

Otro resultado relevante sugiere que una baja integración de los estudiantes a las carreras o instituciones educativas puede constituir un factor de peso al momento de decidir abandonar transitoriamente una institución. Por último, debe señalarse que los temas relativos a la calidad institucional parecen jugar también un función importante en los cambios de institución o programas, especialmente en casos de alumnos con buenos puntajes en las pruebas de selección universitaria. En síntesis, los resultados dan cuenta de una heterogeneidad de condicionantes de la deserción. A continuación, se analizan en detalle las causas que afectan a los desertores temporales del sistema.

\section{Factores explicativos de la deserción temporal}

La deserción temporal contempla situaciones de abandono de estudios universitarios y la reversión posterior de esta situación, ya sea por un cambio de carrera y/o cambio de institución. Los resultados de este 
estudio indican que la falta de claridad vocacional ocupa un lugar central entre los factores explicativos de la deserción temporal de los estudiantes. Los desertores temporales no tenían claramente definida su vocación al momento de ingresar a la universidad. Muchos de ellos tampoco logran definir claramente su vocación una vez que han reingresado al sistema universitario, situación que constituye un factor de riesgo para futuras deserciones, tal como lo sugiere Himmel (2002).

Según lo señalado por los entrevistados, al momento de hacer sus postulaciones a las universidades la mayoría no tenía un claro interés por una carrera específica sino, más bien, un interés general por un área del conocimiento. Las preferencias de los jóvenes no estaban bien formadas cuando debieron definir su futuro educativo, situación que fue transversal a todos los desertores, incluidos los desertores permanentes. Los jóvenes señalaron que no tenían claro qué estudiar porque desconocían o tenían muy poca información sobre las orientaciones de las carreras, los ámbitos de desempeño laboral, los énfasis de los programas de estudios, entre otros aspectos relevantes.

"Opté por sociología sin saber mucho qué era la sociología, y entré y estando en las clases encontré la teoría como muy árida (...) tenía muchos ramos matemáticos que a mí en verdad me cargaban, y los ramos básicos que deberían haberme gustado, a mí no me gustaban..." (Focus Group Nº1, mujer, Ciencias Sociales).

Estos resultados son concordantes con lo planteado por Reay, Davies y Ball (2001), para quienes los procesos de deserción están asociados con el acceso de los estudiantes al sistema educativo terciario y con las oportunidades de elección que tienen al momento de elegir alguna carrera.

Los resultados de esta investigación muestran que los estudiantes eligen una carrera reduciendo una larga lista de posibilidades. Sus 
oportunidades de elección están determinadas por factores sociales, culturales y económicos. En nuestro país, las posibilidades de elección se construyen principalmente sobre la base de los puntajes de la Prueba de Selección Universitaria (PSU) (antes Prueba de Aptitud Académica, PAA), la información y orientación que entregan los pares y padres (redes sociales), para, posteriormente, acudir a fuentes de información "más formales", una vez que han reducido las opciones de selección. En una segunda instancia, factores como la ubicación geográfica y el prestigio de la institución educativa inciden sobre las oportunidades de elección.

Según lo señalado por los entrevistados, el puntaje de la PAA/PSU determina gran parte de la elección. El problema se genera cuando los puntajes obtenidos en estas pruebas son bajos y deben postular a carreras no prioritarias en su escala de preferencias, específicamente por debajo del cuarto o quinto lugar en la lista de postulación. La motivación de los estudiantes, en estos casos, era entrar a la universidad a "estudiar algo", independientemente de sus primeras preferencias. Detrás de esta decisión de ingresar al sistema universitario a estudiar "algo", se observa una fuerte presión social de padres como de los entornos más directos de los estudiantes, situación que es transversal a jóvenes de distintos estratos o grupos sociales. Esta realidad es especialmente significativa en el actual contexto, en que la ES se encuentra en proceso de creciente masificación y es una expectativa y sueño para muchas familias.

Varios de los entrevistados que decidieron ingresar a la universidad a carreras que no estaban dentro de sus principales prioridades lo hicieron con el propósito de cambiarse internamente a otras carreras. Esta situación resultó bastante frecuente entre los desertores temporales.

“...primero, yo entré a la carrera de mala gana, yo entré porque quedé, fue como la sexta opción, quería estudiar Enfermería, esa era mi opción y yo estaba segura que entraba (...) yo quería en otra universidad, acá no, ni siquiera la tomé como opción (...) por los referentes que uno tiene desde afuera de esta universidad (...) entonces, 
la última opción que puse fue Licenciatura en Química aquí, por si acaso, en una de esas, pero nunca pensando que iba a quedar en eso (...) Ya después vino todo lo que es cuando supe que quedé, fue mal, todo el verano mal, mal (...) y (...) empecé yo sola a motivarme, porque ya me matriculé y todo para entrar..." (Focus Group No 5 , mujer, Ciencias Básicas).

Junto con el puntaje de la PAA, otro de los factores que determina fuertemente la decisión de elegir una carrera universitaria se relaciona con la información utilizada para tomar esta decisión. La evidencia recogida muestra que, en la mayoría de los casos, el proceso no resulta ciento por ciento racional y, escasamente, se obtiene o se hace uso de información completa. Por el contrario, la decisión parece responder más bien a intuiciones de los jóvenes o a circunstancias fortuitas del mismo proceso de elección. Un factor común entre los desertores temporales es que ellos utilizaron información limitada para tomar su decisión. En muy pocos casos se hace uso de la información que proveen las fuentes oficiales especializadas en ES (INDICES ${ }^{15}$, por ejemplo); en sólo algunos se hizo uso de Internet para recabar información de las carreras o comparar mallas curriculares.

Ahora bien, sería erróneo plantear que el tema de la elección de carrera sólo depende de la voluntad de los jóvenes para informarse. Claramente, este proceso se ve influenciado por factores de tipo social, familiar y educacional. La decisión de qué carrera estudiar y en dónde está fuertemente vinculada a los capitales sociales y culturales de los estudiantes y sus familias. Los resultados de este estudio, al igual como lo planten Bean y Metzner (1985) y Tinto (1975), muestran que las redes sociales en las que el joven se encuentra inserto actúan como referentes de orientación e información para apoyar su definición vocacional.

La evidencia sugiere que, en los casos en que los capitales culturales y sociales de las familias de los jóvenes son altos, las 
referencias e información acerca de la ES son de mejor calidad. Por el contrario, cuando los padres y redes cercanas a los jóvenes no tienen experiencia de estudios superiores, la información y orientación familiar y social se torna más débil. Esta última situación es especialmente significativa hoy, ya que, según cifras provistas por el INE (2003), más del 70\% de los padres de jóvenes que actualmente están estudiando en la ES no tiene estudios terciarios.

Si bien el tema de la definición vocacional está muy relacionado con el acceso a la educación universitaria (y los factores que restringen sus opciones de elección), los resultados expuestos hasta ahora muestran que esta vinculación no es exclusiva. Los problemas vocacionales no finalizan cuando el estudiante ingresa a la educación universitaria; una vez en la universidad, estos problemas se manifiestan más claramente. Durante el primer y segundo año de estudios los jóvenes experimentan un proceso de "búsqueda", en el que van definiendo con mayor claridad sus intereses vocacionales. En este proceso, muchos de ellos se cambian a otras carreras, programas o instituciones educativas.

En muchos casos, este proceso de búsqueda vocacional no finaliza en estos dos primeros años de estudios. Un grupo importante de jóvenes "busca" por varios años su vocación, lo que les lleva a cursar varias carreras antes de encontrar la que se acerca más a sus intereses. Las consecuencias de estudiar una carrera que no satisface sus expectativas inciden negativamente en su motivación académica. La baja motivación se manifiesta en conductas tales como, la pérdida de interés por ir a clases, inasistencia a pruebas, bajo rendimiento, entre otros aspectos. Estos resultados son concordantes con lo planteado por Reed (1968), quien destaca la relevancia de los factores motivacionales en el proceso de deserción temporal y, específicamente, su incidencia en los rendimientos académicos.

Adicionalmente, el rendimiento académico pareciera estar muy vinculado a factores socioculturales. Según lo señalado por los desertores temporales, el principal problema que debieron enfrentar 
cuando ingresaron a estudiar en la universidad fue adaptarse al ritmo universitario y desarrollar hábitos permanentes de trabajo y de estudio. La evidencia sugiere que el ingreso a la educación superior significa un salto cualitativo para los jóvenes respecto de la educación media y que, en muchos casos, ellos no estaban preparados para enfrentar las exigencias universitarias. En particular, este tipo de problemas fue manifestado en mayor medida por alumnos provenientes de colegios particulares subvencionados y municipales.

“... en primer año no me fue mal, pero no le tomé el peso (...) aparte que venía con el ritmo del colegio, que era súper fácil (...) entonces yo tuve algunos problemas (...) pero después ahí con la ayuda de los compañeros, cuando me juntaba a estudiar con ellos ahí me empecé a sacar buenas notas (...) pero en lo educacional no llegué con mala base, si prácticamente los pasé bien, arriba del 5,5 , excepto uno que fue por la primera prueba que me lo bajó caleta..." (Focus Group N² 2, hombre, Tecnología).

La falta o problemas de hábitos de estudio pudiera vincularse con un tema de (en palabras de Bourdieu) habitus de los estudiantes. Las motivaciones y disposiciones de los estudiantes hacia la educación universitaria y sus metas futuras no están suficientemente asentadas cuando ingresan a la ES. Para muchos jóvenes, especialmente para aquellos que no corresponden con el perfil "tradicional" de alumnos universitarios, el proceso de ajuste a la vida académica resulta complejo y dificultoso en una primera etapa. Coincidentemente con lo planteado por Reed (1968), quien señala que algunas de las variables que inciden en la motivación del alumnado y, por ende, en su desempeño académico son: el sentido que el alumno asigna a las tareas académicas, la relevancia de los estudios con relación a sus metas futuras, el desafío académico que supone la carrera, entre otros aspectos.

Muy relacionado con el tema de las disposiciones culturales hacia la educación, aparece el de las expectativas laborales y profesionales que tienen los jóvenes sobre sus carreras. La mayoría de los entrevistados manifestó que sólo dentro de la universidad 
conocieron las reales oportunidades y perspectivas laborales de las carreras a las que ingresaron. Una vez dentro, y cuando percibieron una falta de proyección en una profesión o disciplina, la decisión de desertar se hizo patente. Es interesante notar que todos los entrevistados que señalaron que sus expectativas profesionales y laborales no se habían cumplido, desconocían al momento de ingresar a la universidad qué hacían los profesionales egresados de estas carreras y en qué áreas trabajaban. En muy pocos casos los entrevistados averiguaron previamente cuál era el ingreso promedio que recibían los egresados de esas carreras, el campo laboral de desempeño u otros aspectos. Este hecho reafirma la escasa información y orientación con que cuentan los jóvenes al momento de ingresar a diferentes programas educativos.

Es sólo dentro de la universidad, particularmente durante el primer año de sus estudios, donde los jóvenes adquieren un conocimiento más concreto de las posibilidades que ofrecen las carreras. Esta situación es especialmente importante en aquellas que no tienen una clara orientación profesional (filosofía, por ejemplo) o cuentan con un mercado de trabajo poco conocido o desarrollado.

“¿en qué voy a trabajar, qué voy a hacer después? (...) Entonces fui a preguntar qué se hace como sociólogo, fui a empresas, había gente que trabajaba en publicidad, otra en marketing, otros hacían investigaciones, otros se dedicaban solamente a estadísticas, casi como un ingeniero estadístico, porque no le quedaba otra que hacer (...) esa fue por lo menos la situación que yo vi. (...) y eso me produjo mucha inseguridad en lo que iba a hacer como sociólogo..." (Focus Group № 1, hombre, Ciencias Sociales).

En síntesis, los resultados muestran la importancia de los factores socioculturales, vocacionales y motivacionales en la deserción universitaria. Para un grupo importante de desertores temporales, la decisión de abandonar una carrera tiene directa relación con problemas vocacionales y con las expectativas laborales que tienen respecto de esa 
carrera. Por otra parte, los resultados también muestran la significativa incidencia de los factores socioeconómicos y culturales en los procesos de ingreso y deserción. Las restricciones y oportunidades que enfrentan los estudiantes al momento de ingresar al sistema determinan, en gran medida, su permanencia y movilidad dentro de éste. Si bien estos resultados no pueden ser considerados concluyentes, dado el carácter exploratorio del estudio, plantean varias preguntas e hipótesis que pueden ser estudiadas en futuras investigaciones. A continuación, pasaremos a revisar en detalle las causas de la deserción universitaria permanente. Los resultados muestran un patrón explicativo distinto al de la deserción temporal.

\section{Factores explicativos de la deserción permanente}

El abandono permanente de la educación universitaria forma parte de un proceso de deserciones sucesivas dentro del sistema de educación superior. Nueve de los doce desertores permanentes entrevistados cursaron dos o más carreras antes de tomar la decisión de abandonar la educación superior de modo definitivo.

En relación con las causas que originaron la deserción definitiva del sistema universitario, los factores no académicos resultaron ser especialmente determinantes. Sin embargo, a diferencia de los desertores temporales, una proporción importante de los permanentes señaló haber abandonado la universidad por causas económicas y familiares. Circunstancias tales como la pérdida del empleo de algún familiar directo, o bien que el estudiante debió asumir responsabilidades como jefe de familia se constituyeron en factores fundamentales para abandonar permanentemente el sistema educativo. En estos casos, aunque puede ser entendido como voluntario -ya que no es la institución la que los expulsa- no constituye una deserción buscada o deseada por los estudiantes. Por el contrario, la decisión de dejar de estudiar es percibida como difícil debido a que existen expectativas familiares y personales respecto de la ES. Los desertores entrevistados señalaron que una de las razones más importantes por la cual desearían retomar o iniciar nuevos estudios se relaciona con 
los beneficios asociados a la obtención de un grado académico o título profesional.

"Yo siempre dispuse de mi plata para mí, independiente de que hiciera o no hiciera, además tenía la ayuda de mi papá, que mi papá tenía sus ingresos extras. Entonces, cuando se murió, mi mamá se quedó con una pensión y sin ningún peso extra. Mi papá era bien cómplice en eso, plata no me faltaba, y fue súper fuerte encontrarse con el cambio de rol, ahora tenía que sostener". (Entrevista $\mathrm{N}^{\circ}$ 2, mujer, Ciencias Sociales).

Para un grupo significativo de desertores permanentes los factores económicos están fuertemente asociados a cambios en sus condiciones familiares. De los doce entrevistados, siete indicaron que los cambios en su situación familiar habían dificultado continuar con sus estudios superiores. La evidencia sugiere que cambios en la estructura y composición familiar generan cambios significativos en términos de bienestar económico para las familias. La combinación de responsabilidades familiares y económicas dificulta la continuación de estudios universitarios, en particular para aquellos estudiantes que no son "tradicionales" en el sistema educativo. El nacimiento de un hijo o la muerte del padre o sostenedor familiar fueron los condicionantes que impidieron continuar en la universidad. Bean y Metzner (1985), coincidentemente, destacan la importancia de variables relativas al cambio en responsabilidades familiares como aspectos detonantes de la deserción.

"Me tuve que venir a trabajar a Santiago. Yo estaba trabajando allá [ciudad], trabajaba en un banco. Vino la crisis asiática y quedé con el puro sueldo. Teníamos un montón de gastos, entonces con mi sueldo pagaba la universidad y quedaba mirando la carnicería. En ese minuto además mi papá murió, yo me tuve que hacer cargo de mi casa y en ese minuto me ofrecieron pega acá, me pagaban el doble de lo que me pagaban allá y me vine". (Entrevista $N^{\circ} 10$, mujer, Administración y Comercio). 
Los factores socioeconómicos referidos dan cuenta de la vulnerabilidad de diversos grupos de estudiantes en la educación universitaria actual. Ellos no cuentan con una estructura de protección que alivie el efecto de estas transiciones o cambios demográficos en sus vidas. La gran mayoría de estos desertores mencionaron no haber contado con ayuda institucional -a través de becas o préstamos- que permitiera aliviar su precaria situación financiera. Esta situación debiera ser abordada o, al menos, considerada por las instituciones educativas y también por las políticas gubernamentales de educación superior. Gran parte de los beneficios estatales entregados en la actualidad se concentran en el acceso y no en la permanencia en el sistema.

Los desertores permanentes manifestaron también la carencia de hábitos de estudios, disciplina y competencias, y una baja identificación con sus carreras como aspectos que determinaron su rendimiento y motivación académica en la universidad. Dado que muchos de ellos estaban afectados por situaciones de mayor vulnerabilidad, las posibilidades de continuar estudiando se hicieron imposibles.

"Cuando entré a la universidad mis compañeros traían hábitos de estudios creados, a mí me fallaba el inglés. Mi colegio no tenía mayor exigencia. En el segundo año de universidad me ofrecieron expresión oral y clases de inglés". (Entrevista No 1 , hombre, Derecho).

Los desertores permanentes, al igual que los temporales, tomaron la decisión de estudiar sin demasiada certeza e información. Es importante destacar la falta de orientación y apoyo general durante el proceso de elección de carreras. De acuerdo con lo señalado por los entrevistados, el colegio o liceo proporcionó recursos muy limitados para orientar su proceso de definición vocacional; en muy pocos casos los establecimientos entregaron información o guía respecto de las carreras. Las familias, por su parte, tampoco juegan un rol activo en este proceso. Adicionalmente, algunos entrevistados indicaron que las universidades dificultaban el proceso de selección al promocionar de manera excesiva las cualidades de sus carreras. 
"... Cuando tú vas saliendo del colegio no sabes nada, así que te comes cualquier cosa que te vendan." (Entrevista $\mathrm{N}^{\circ} 7$, mujer, Humanidades).

Los resultados presentados en este apartado muestran que la situación de los desertores permanentes puede calificarse como vulnerable por las condiciones económicas y familiares que los aquejan. A diferencia de los desertores temporales, este grupo no se encuentra en proceso de "búsqueda vocacional". El hecho de abandonar la ES constituye para este grupo en particular una pérdida de oportunidades sociales y económicas significativa.

\section{Conclusiones}

Los resultados de este estudio confirman lo planteado por investigaciones internacionales y nacionales sobre la multiplicidad de factores de distinta índole que explican la deserción universitaria. La naturaleza variada de factores explicativos va configurando distintas formas de deserción. En nuestro estudio se destacan la deserción temporal y la deserción permanente, las cuales responden a patrones explicativos distintos.

El estudio sugiere que, entre la gran variedad de factores explicativos de la deserción universitaria, los factores no académicos son muy relevantes a la hora de explicar este fenómeno. Por el contrario, este estudio no pudo recabar suficientes antecedentes acerca del carácter voluntario o involuntario de la deserción, y de la relevancia de este atributo al momento de definir formas o tipos de deserción universitaria.

Entre los factores no académicos que resultaron relevantes para explicar situaciones de abandono temporal y permanente se encuentran aspectos de tipo vocacional, motivacional y socioeconómico. La falta de claridad vocacional resultó ser uno de los factores más mencionados para explicar la deserción temporal de los jóvenes universitarios. A su vez, la vocación juega un rol importante pero no preponderante en la deserción permanente. 
La evidencia encontrada también sugiere que los problemas vocacionales están fuertemente relacionados con el proceso de elección de carrera (ingreso a la educación universitaria) y con las expectativas laborales que los jóvenes tienen sobre ésta. Al momento de tomar la decisión de ingresar a la educación superior los jóvenes desconocen o cuentan con escasa información sobre lo que significa efectivamente estudiar una carrera universitaria. Esta situación lleva a que tomen decisiones inconsistentes. Por otra parte, las preferencias de muchos jóvenes desertores están poco formadas y vinculadas a un área general del conocimiento más que una carrera específica.

Al momento de seleccionar una carrera o universidad, los grupos de referencia (pares), la información dispersa y otras contingencias resultan ser tan importantes como el análisis de mallas curriculares, las búsquedas de información en Internet y las visitas a universidades. En este contexto, parece pertinente proponer que los jóvenes sean expuestos a múltiples experiencias e información que les permita conocer tanto los desafíos cognitivos asociados a las carreras, como el medio profesional y las remuneraciones y oportunidades que éstas y las instituciones ofrecen.

El proceso de búsqueda y definición vocacional no finaliza cuando los jóvenes ingresan a la universidad. Por el contrario, en muchos casos se refuerza una vez los estudiantes se encuentran en ella. En las universidades los jóvenes descubren cuáles son los campos de desempeño profesional real y los retornos económicos de sus carreras, situación que, en muchos casos, los lleva a replantearse respecto de su decisión inicial de estudio. Un grupo significativo de desertores temporales entrevistados se cambió de carrera porque ésta no correspondía a las expectativas laborales y económicas esperadas.

La tarea de información debiera ser compartida por familias, escuelas y universidades. Idealmente, la definición de un área vocacional debiera desarrollarse desde la educación primaria, con el fin de garantizar una elección con mayor información y certeza por parte de los jóvenes. Muchos jóvenes se ven enfrentados a la 
elección de una carrera con escasa información y bastante presión familiar y social. Más aún, el tiempo con que cuentan para tomar la decisión -posterior a la obtención de los puntajes de las pruebas de selección-resulta especialmente apremiante en los casos de aquellos que postulan a las universidades del Consejo de Rectores.

Las familias y escuelas podrían jugar un rol más activo proporcionando información y orientando la decisión sobre carreras y universidades. Sin embargo, se reconoce que muchas no pueden cumplir con esta labor, ya que carecen de los recursos o capitales sociales para desarrollarla de forma activa. Por esta razón, el sistema educacional debe jugar un rol más preponderante. Experiencias como visitas guiadas a universidades, presentaciones de profesionales sobre las actividades que realizan y de profesores universitarios en escuelas y liceos podrían ser de gran utilidad. En otros países, tales como Estados Unidos y Australia, existen asignaturas específicas que orientan la decisión profesional.

Con relación a la deserción permanente, podemos señalar que los factores socioeconómicos son especialmente relevantes. El nacimiento de hijos, matrimonio, muerte o enfermedad del jefe o sostenedor de la familia son factores importantes que desencadenan la deserción definitiva del sistema. Estos cambios tienen un impacto directo en términos del bienestar económico del estudiante, ya que alteran substantivamente la estructura presupuestaria familiar, dificultando la posibilidad de estudiar en el corto y mediano plazo. En estos casos, la decisión de retomar los estudios puede hacerse en extremo dificultosa, ya que la vulnerabilidad puede persistir por un periodo significativo de tiempo, situación que desalienta a retomar los estudios.

La combinación de responsabilidades familiares y económicas obstruye la continuación de estudios universitarios, en particular para aquellos estudiantes que no son "tradicionales" en el sistema educativo. Los problemas descritos dan cuenta de la vulnerabilidad de muchos estudiantes que se ven forzados a emigrar de la 
universidad porque no pueden responder a las exigencias de las instituciones educativas. Lamentablemente, en estos casos de mayor vulnerabilidad, existen pocos resguardos financieros que permitan moderar los efectos de los ciclos familiares y financieros, situación que hace de la deserción un problema social significativo. Considerando que los retornos de la educación universitaria son en promedio positivos, podrían diseñarse créditos acotados que permitieran enfrentar contingencias familiares específicas, ya que éstas pueden alterar de manera determinante y positiva el bienestar futuro de jóvenes con mayor vulnerabilidad.

Finalmente, en relación con la incidencia de factores académicos, se aborda brevemente el problema de la calidad de las instituciones universitarias. Este aspecto resultó relevante en los casos de desertores temporales, especialmente en los casos de alumnos con buenos puntajes en las pruebas de selección universitaria. Éstos argumentaron que cuando las instituciones a las que ingresaron no cumplían con sus expectativas académicas -en especial en relación con infraestructura, calidad del profesorado o mallas curriculares- optaron por cambiarse a instituciones que, ellos pensaban, tenían mayor rigor académico.

Aunque este grupo -con altos puntajes y altamente motivados al aprendizaje- no constituyó un grupo mayoritario entre los entrevistados, constituye un segmento particular o diferenciado que debe ser considerado como potencialmente desertor y al que, por tanto, deben orientarse estrategias específicas para asegurar su permanencia en el sistema.

Instituciones de regular calidad tienen dificultades para satisfacer las expectativas académicas de muchos estudiantes y, particularmente, de aquellos motivados y orientados al logro que buscan desarrollar sus capacidades y habilidades en el sistema educativo. Por lo tanto, esta evidencia nos sugiere que las universidades no están exentas de responsabilidad en la deserción estudiantil y deben comprender este problema como un asunto en el que también está en juego su integridad y prestigio institucional. 


\section{Referencias bibliográficas}

Baker, T. \& Velez, W. (1996) Access to and Opportunity in Postsecondary Education in United States; A Review. Sociology Of Education, 69, 82101.

Bank, B., Slaving, R. \& Biddke, B. (1990) Effects of Peer, Faculty and Parental Influences on Students' Persistence. Sociology Of Education, 63, 208225.

Bayer, A. (1968) The College Drop-Out: Factors Affecting Senior College Completion. Sociology Of Education, 41, 305-316.

Bean, J. \& Metzner, B. (1985) A Conceptual Model of Nontraditional Undergraduate Student Attrition. Review Of Educational Research, 55 , 485-540.

Bourdieu, P. (1986) The Forms of Capital In: G Richardson, Handbook of Theory and Research for Sociology of Education. New Cork: Greenwood Press.

Bourdieu, P. Passeron, J.C. (1973) Los Estudiantes y la Cultura, Buenos Aires: Labor.

Bravo, D. \& Contreras, D. (2000) Competencias Básicas de la Población Adulta. Santiago de Chile: Departamento de Economía, Universidad de Chile.

Bravo, D. \& Manzi, J. (2002) Equidad y Resultados Educacionales: SIMCE y PAA. Santiago de Chile, Departamento de Economía, Universidad de Chile \& Escuela de Psicología, Pontificia Universidad Católica de Chile.

Braxton, J., Johnson, R. M. \& Shaw-Sullivan, A. (1997) Appraising Tinto's Theory of College Student Departure. In: Smart J.C. Higher Education Handbook of Theory and Research. New York: Agathon Press.

Brunner, J. J. (2005) Guiar el Mercado. Informe sobre la Educación Superior en Chile. Santiago de Chile.

Brunner, J. J. \& Elaqua, G. (2003) Informe de Capital Humano, Santiago de Chile.

Brunner, J. J. \& Meller, P. (2004) Oferta y Demanda de Profesionales y Técnicos en Chile. Santiago de Chile.

CADEM (1996) Informe al Consejo Superior de Educación sobre los resultados de los focus groups realizados con estudiantes secundarios. Santiago de Chile, Mimeo. 
Camburn, E. (1990) College Completion Among Students from High Schools Located in Large Metropolitan Areas. American Journal of Education, 98, 551-569.

CIDE (2001) III Encuesta a Actores del Sistema Educativo. Santiago de Chile: Centro de Investigación y Desarrollo de la Educación.

CIDE (2003) IV Encuesta a Actores del Sistema Educativo. Santiago de Chile: Centro de Investigación y Desarrollo de la Educación.

Clark, B. (1960) The Cooling-Out Function in Higher Education. The American Journal of Sociology, 65, 569-576.

Cowhig, J. (1963) Who Do They Leave College? The School Review, 71, 330336.

Consejo Superior de Educación (2006) INDICES 2006.

Consejo Superior de Educación (2007) INDICES 2007.

Delannoy, F. (2000) Education Reforms in Chile, 1980-98: A Lesson in Pragmatism. Education Reform and Management Publication Series. Washington DC: World Bank.

Dohn, H. (1991) Drop-Out in The Danish High School (Gymnasium): An Investigation of Psychological, Sociological and Pedagogical Factors. International Review of Education, 37, 415-428.

Durkheim, E. (1951) Suicide: A Study in Sociology. Glencoe, New York: The Free Press.

Ehrenberg, R. \& Sherman, D. (1987) Employment While in College, Academic Achievement, and Post College Outcomes: A Summary of Results. The Journal of Human Resources, 22, 1-23.

Fielding, A., Belfield, C. \& Thomas, R. (1998) The Consequences of DropOuts on the Cost-Effectiveness of 16-19 Colleges. Oxford Review of Education, 24, 487-511.

González, L. E. \& Uribe, D. (2003) Estimaciones sobre la repitencia y deserción en la educación superior. Revista Calidad en la Educación, 19, 90-108.

González, L. E., Uribe, D. \& González, S. (2005) Estudio sobre la repitencia y deserción en la educación superior chilena. Instituto Internacional para la Educación Superior en América Latina y el Caribe, IESALCUNESCO. 
Hackmann, R. \& Dysinger, W. (1970) Commitment to College as a Factor in Student Attrition. Sociology of Education, 43, 311-324.

Himmel, E. (2002) Modelos de Análisis de la Deserción Estudiantil en la Educación Superior. Revista Calidad en La Educación, 17, 75-90.

Ine (2003) Cuánto y cómo cambiamos los chilenos: balance de una década 1992-2002, Santiago de Chile, Instituto Nacional de Estadísticas, Publicaciones del Bicentenario.

Kamens, D. (1971) The College Charter and College Size: Effects and Occupational Choice and College Attrition. Sociology of Education, 44, 270-296.

Larrañaga, O. (2002) Elementos para una reforma del sistema de crédito estudiantil en Chile. Facultad de Economía y Administración, Universidad de Chile, Documento de Trabajo Nº 189.

Light, A. \& Strayer, W. (2000) Determinants of College Completion: School Quality or Student Ability? The Journal of Human Resources, 35, 299- 332.

MINEDUC (2005) Compendio de Educación Superior 2005.

Mizala, A. \& Romaguera, P. (2004) Remuneraciones y tasas de retorno de los profesionales chilenos. In Brunner, J. J. \& Meller, P. (Eds.) Oferta y demanda de profesionales y técnicos en Chile: El rol de la información pública. Santiago de Chile, Ril Editores.

OECD (1998) Education at Glance. OECD Indicators 1998. Paris: Organization for Economic Cooperation and Development.

OECD (2000) Literacy in the Information Age. Paris, Organization for Economic Cooperation and Development.

OECD (2002) Education at Glance. OECD Indicators 2002. Paris, Organization for Economic Cooperation and Development.

OECD (2002) Financing Education-Investments and Returns, Analysis of the World Education Indicators 2002 Edition. Paris, Organization for Economic Cooperation and Development.

OECD (2004) Revisión de politicas nacionales de educación en Chile. Paris, Organization for Economic Cooperation and Development.

OECD (2005) Education at Glance. OECD Indicators 2005. Paris, Organization for Economic Cooperation and Development.

Oedel, P. \& Zemsky, R. (1983) The Structure of College Choice. New York. 
PNUD (2005) Expansión de la educación superior en Chile: hacia un nuevo enfoque de la equidad y calidad, PNUD: Temas de Desarrollo Humano Sustentable \# 10.

Psacharopoulos, G. \& Patrinos, H. A. (2002) Returns to Investment in Education: A Further Update. World Bank.

Psacharopoulos, G. \& Patrinos, H. A. (2004) Returns to Investment in Education: A Further Update. Education Economics, 12.

Reay, D., Davies, J., David, M. \& Ball, S. (2001) Choices of Degree or Degrees of Choice? Class, Race and the Higher Education Choice Process. Sociology, 35, 855-874.

Reed, H. (1968) College Students Motivations Related to Voluntary Dropout and Under-Overachievement. Journal of Educational Research, 61.

Smith, J. \& Naylor, R. (2001) Dropping Out of University: A Statistical Analysis of the Probability of Withdrawal For UK University Students. Journal of the Royal Statistical Society, 164, 389-405.

Smith, T. (1982) College Dropouts: An Analysis of the Psychological WellBeing and Attitudes of Various Educational Groups. Social Psychology Quarterly, 45, 50-53.

Spady, W. G. (1970) Dropouts from Higher Education: An Interdisciplinary Review and Synthesis. Interchange, 1, 64-85.

Tierney, W. (1999) Models of Minority College-Going and Retention: Cultural Integrity versus Cultural Suicide. The Journal of Negro Education, 68, 80-91.

Tillman, S., C.A (2002) Barriers to Student Persistence in Higher Education.

Tinto, V. (1975) Dropout From Higher Education: A Theoretical Synthesis of Recent Research. Review of Educational Research, 45, 89-125.

UNESCO (1997) Clasificación internacional normalizada de la educación. París: UNESCO.

Worldbank (2002) World Development Indicators 2002. World Bank.

Worldbank (2005) World Development Indicators 2005. Washington DC.

Recibido: 13 de abril de 2007

Aceptado: 26 de abril de 2007 